\title{
Shape and topology optimization based on the convected level set method
}

\author{
Kentaro Yaji ${ }^{1}$ - Masaki Otomori ${ }^{2}$ Takayuki Yamada ${ }^{1} \cdot$ Kazuhiro Izui $^{1}$. \\ Shinji Nishiwaki ${ }^{1} \cdot$ Olivier Pironneau $^{3}$
}

Received: 30 June 2015 / Revised: 1 February 2016 / Accepted: 6 March 2016 / Published online: 22 April 2016

(C) Springer-Verlag Berlin Heidelberg 2016

\begin{abstract}
The aim of this research is to construct a shape optimization method based on the convected level set method, in which the level set function is defined as a truncated smooth function obtained by using a sinus filter based on a hyperbolic tangent function. The local property of the hyperbolic tangent function dramatically reduces the generation of red the error between the specified profile of the hyperbolic tangent function and the level set function that is updated using a time evolution equation. In addition, the small size of the error facilitates the use of convective reinitialization, whose basic idea is that the reinitialization is embedded in the time evolution equation, whereas such treatment is typically conducted in a separate calculation in conventional level set methods. The convected level set method can completely avoid the need for additional calculations when performing reinitialization. The validity and effectiveness of our presented method are tested with a mean compliance minimization problem and a problem for the design of a compliant mechanism.
\end{abstract}

Kentaro Yaji

yaji.kentarou.74v@st.kyoto-u.jp

1 Department of Mechanical Engineering and Science, Graduate School of Engineering, Kyoto University, Kyotodaigaku-katsura, Nishikyo-ku, Kyoto 615-8540, Japan

2 AISIN AW Co., LTD. Fujii-cho, Takane 10, Anjo, Aichi, 444-1192, Japan

3 Sorbonne Universités, UPMC Univ Paris 06, UMR 7598, Laboratoire Jacques-Louis Lions (LJLL),Boite courrier 187, 75252 Paris Cedex 05, France
Keywords Shape optimization · Hyperbolic tangent level set function - Convective reinitialization .

Shape sensitivity $\cdot$ Mesh adaptation

\section{Introduction}

The study of optimal shape design under various specified conditions is an attractive area of research for engineers and mathematicians. Actually, there are many different kinds of numerical methods applied to various optimal shape design problems.

Shape optimization is one of the well-studied structural optimization methods, and its key idea is to optimize a performance, defined as the objective function, by moving structural boundaries (Pironneau 1984; Sokolowski and Zolesio 1992). Topology optimization has also been an attractive structural optimization method since Bendsøe and Kikuchi (1988) first proposed the so-called homogenization design method. The basic idea of topology optimization is the introduction of an extended design domain and the replacement of the optimization problem with a material distribution problem using the characteristic function. Topology optimization allows the creation of new boundaries in the design domain during the optimization process, which is not possible with classical shape optimization. Recent developments in the field of topology optimization have been categorized in a review paper by Sigmund and Maute (2013).

The level set method for structural optimization is another popular approach, since Osher and Sethian (1988) constructed a fundamental methodology for tracking fronts and free boundaries. The novel aspect of the use of a level set method for structural optimization is that the shape 
boundaries can split or merge during the optimization in an Eulerian coordinate system, whereas such topological changes are typically inhibited in classical shape optimization methods based on a Lagrangian coordinate system. Additionally, although this approach essentially does not allow the creation of new boundaries in the design domain during the optimization process, expanded grayscale areas can be eliminated. Thus, areas of the design domain in topology optimization approaches that have intermediate material density and are therefore generally meaningless from an engineering standpoint, can be eliminated in level set-based approaches, because the structural boundaries are represented as the iso-surface of a scalar function, the so-called level set function.

For these reasons, level set-based approaches have been often categorized as topology optimization methods that are free from grayscales. On the other hand, there is another way of classifying structural optimization methods, based on how the design sensitivity is handled, in contrast to the above classification that focuses on whether or not topological changes are allowed. In this classification, level set-based approaches are categorized as shape optimization methods because the shape sensitivity is used to control the structural boundaries. As will be described later, since our methodology is mainly based on previous studies proposed by Allaire et al. (2004, 2014), who argue that their level set-based approach is categorized as a shape optimization method due to the way the shape sensitivity is handled, we adopt their position in this paper. Seminal research pertaining to the level set method for structural optimization can be found in (Sethian and Wiegmann 2000; Wang et al. 2003; Allaire et al. 2004), and the reader is referred to a recent monograph by Van Dijk et al. (2013) in which a variety of structural optimization methods based on the level set method are discussed.

In conventional approaches based on the level set method, the level set function is evolved using an advection equation, the so-called Hamilton-Jacobi equation, during the optimization process. This equation is used to capture the structural boundary, and the convection velocity is typically defined using the shape derivative. Many previous studies have applied structural optimization based on the level set method to a variety of optimization problems, such as a stiffness maximization problem for linear and nonlinear elastic structures (Allaire et al. 2004; Luo and Tong 2008), a shell structure design problem (Park and Youn 2008), and a multi-material design problem (Wang and Wang 2004; Luo et al. 2009). In addition, level set-based approaches have been applied to other physical problems such as fluid problems (Amstutz and Andrä 2006; Challis and Guest 2009), and electromagnetic problems (Khalil et al. 2010; Zhou et al. 2010).
However, conventional approaches based on the level set method still have serious drawbacks. One particularly troublesome problem is that since the approach is based on the concept of shape optimization, the optimal shape is strongly dependent on the initial design setting. Another major problem is that since the level set function is typically defined as a signed distance function whose profile must be maintained, the gradient of the level set function must be adjusted to assume a constant value across the entire design domain, by incorporating a reinitialization procedure. Thus, an additional time evolution equation, i.e., the so-called unsteady Eikonal equation, must be periodically solved to ensure the stability of the Hamilton-Jacobi equation calculations. This is because the level set function may become too steep or too flat if the Hamilton-Jacobi equation is solved alone.

Allaire et al. (2005) proposed a methodology based on the concept of the bubble method (Eschenauer et al. 1994) incorporating a topological derivative (Sokolowski and Zochowski 1999) that enables the creation of new boundaries in the design domain during the optimization process. The advantage of this approach is that since it is unnecessary to include holes corresponding to the void domain in the initial design, the dependency of optimal shapes with respect to the initial design settings can be mostly avoided. This approach has been applied to various optimization problems such as a stress minimization problem (Allaire and Jouve 2008), and fluid problems (Amstutz and Andrä 2006; Challis and Guest 2009).

Radical methods that do without reinitialization have been developed (Wei and Wang 2009; Yamada et al. 2010), where the regularization term is introduced in the objective functional to preserve the smoothness of the level set function, redefined as a piecewise constant function that is originally based on the concept of the phase field method (Cahn and Hilliard 1958; Allen and Cahn 1979). In the approaches dealing with this piecewise constant function, the implicit boundary expressions based on the level set function are retained, and neither the Hamilton-Jacobi equation nor the unsteady Eikonal equation need to be solved during the optimization process. Although these methods enable the optimization process to proceed without reinitialization, a regularization term, typically defined as the gradient of the level set function, must be included in the objective functional. Based on the classical level set-based approach that deals with a signed distance function, Duan et al. (2008) and Zhu et al. (2015) proposed methodologies incorporating a regularization term that maintains the profile of the level set function as a signed distance function. However, a significant problem remains in the above methodologies, that of determining an appropriate value for the tuning parameter used to control the degree of regularization. 
On the other hand, Wang and Wang (2006) proposed a level set-based approach, in which a radial basis function (RBF) is used to express the structural boundary, and demonstrated that it is possible to conduct the reinitialization procedure without the use of an additional term in the objective functional. In their approach, coefficients of RBFs correspond to the design variables, while in most level setbased approaches, local nodal values are used as the design variables in the discrete formulation. This approach may lead to a rapid rate of shape change, but requires additional memory and computations due to the interaction of the basis functions. As an alternate approach that is able to not only avoid the reinitialization treatment but also reduce the computational cost, Guo et al. (2014) have recently proposed a unique methodology, in which the aim of the optimization problem is to design the layout of 'morphable' components whose boundaries are explicitly represented using the level set function. Due to the use of only a few design variables, the computational cost in this approach is small.

In the field of computational fluid dynamics dealing with two-phase fluid flows, Ville et al. (2011) proposed a new approach, the so-called convected level set method, in which the level set function is evolved while maintaining its profile, by incorporating an additional term in the advection equation, a numerical scheme called convective reinitialization. Additionally, since only the information in the vicinity of the interface between the phases in a two-phase fluid flow is necessary, the signed distance function is replaced by a local function defined as a truncated smooth function that is obtained with a sinus filter. This local function is constant almost everywhere except in the vicinity of the interface, and corrective measures to maintain its profile are therefore less necessary compared with implementations using the original signed distance function. That is, the attractive feature of the convected level set method is the introduction of the filtered level set function, which preserves its profile as a smooth truncated function without the need for additional reinitialization calculations. Although the convected level set method has not yet been applied to a structural optimization problem, it is obvious that it is a promising approach for shape optimizations. Furthermore, a regularization term in the objective functional is not needed when using the convected level set method for shape optimizations, whereas even in the new approaches mentioned above, the reinitialization procedure cannot be avoided without the use of an additional regularization term. Although the convected level set method requires an additional term in the advection equation, the degree of reinitialization can be appropriately adjusted using a coefficient of the additional term, whose value can be estimated from the time and space step settings for the discretized advection equation (Ville et al. 2011).
This paper presents a shape optimization method based on the convected level set method. The proposed method extends a methodology (Allaire et al. 2004) in which the level set function is defined as a signed distance function and evolved based on the information of a shape derivative using the Hamilton-Jacobi equation, which requires a reinitialization procedure. The novel aspect of our proposed method is the introduction of the sinus filter and convective reinitialization for the truncated smooth level set function.

The remainder of this paper is organized as follows. In Section 2, the model problem in this study is briefly discussed. In Section 3, the conventional level set method is introduced, and then the details of the convected level set method are explained, including the definition of the sinus filer as a hyperbolic tangent function (Coupez et al. 2015). Section 4 presents the numerical implementation of the proposed method, and we also indicate the utility of a mesh adaptation technique that increases the computational accuracy of the time evolution equation for the hyperbolic tangent level set function. Several numerical examples for the mean compliance minimization problem and the complaint mechanism problem are provided to confirm the validity and utility of the proposed method in Section 5. Finally, Section 6 concludes this paper and summarizes the obtained results.

\section{Formulation of the optimization problem}

We consider a model problem for a linearized elastic structure. Let $\Omega \subset \mathbb{R}^{d}(d=2,3)$ be filled with a linearly elastic isotropic material. The boundary of $\Omega$ is defined as a Dirichlet boundary, $\Gamma_{D}$, on which the displacement $\mathbf{u}$ is fixed, and a Neumann boundary exists at $\Gamma_{N}$ where a traction $\overline{\mathbf{t}}$ is applied. The displacement field in $\Omega$ is the solution of the following boundary value problem,

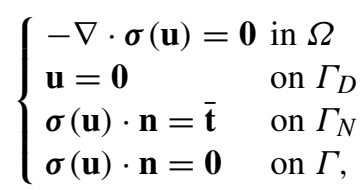

where $\Gamma=\partial \Omega \backslash\left(\Gamma_{D} \cup \Gamma_{N}\right)$ is the traction-free boundary and $\mathbf{n}$ is the outer unit normal to $\partial \Omega$. In addition, $\boldsymbol{\sigma}=\mathbf{C}: \boldsymbol{\epsilon}$ represents the stress tensor, in which $\mathbf{C}$ and $\boldsymbol{\epsilon}=\left(\nabla \mathbf{u}+(\nabla \mathbf{u})^{\mathrm{T}}\right) / 2$ are the elastic tensor and the linearized strain tensor, respectively. The above boundary value problem (1) has a unique solution in $\mathbf{u} \in H^{1}(\Omega)^{d}$ when $\Gamma_{D}$ is non empty. 
For shape optimization, we consider the following minimization problem with respect to the objective functional $J(\Omega)$ :

$\inf _{\Omega \in \mathscr{U} \mathrm{ad}} J(\Omega)=\int_{\Omega} j(\mathbf{u}) \mathrm{d} \Omega+\int_{\Gamma_{N}} k(\mathbf{u}) \mathrm{d} \Gamma$,

where $j, k: \mathbb{R}^{d} \rightarrow \mathbb{R}$ are smooth functions and $\mathscr{U}_{\text {ad }}$ is a set of admissible shapes. The specific definitions of $J(\Omega)$ will be discussed in Section 5. Since $\Omega$ varies during the optimization process, we introduce a working domain $D$, which is a bounded open set of $\mathbb{R}^{d}$ and contains all admissible shapes of $\Omega$. Thus, the set $\mathscr{U}_{\text {ad }}$ in this study is defined as follows:

$\mathscr{U}_{\mathrm{ad}}=\left\{\Omega \subset D\right.$ such that $\left.|\Omega|=V, \Gamma_{D} \cup \Gamma_{N} \subset \partial D\right\}$,

where $V$ represents the prescribed volume of the material domain. The schematic diagram of the working domain $D$ including the material domain $\Omega$ with the boundary settings is shown in Fig. 1.

With the help of Lagrange multipliers or penalty and regularization coefficients, a constrained optimization problem is typically replaced with an unconstrained optimization problem. Introducing a regularization coefficient $l$ for the prescribed volume constraint, we consider the following minimization problem,

$\inf _{\Omega \in \mathscr{U} \text { ad }} L(\Omega)=J(\Omega)+l|\Omega|$.

The aim of this study is to minimize the objective functional $J$ by varying the shape of $\Omega$. Thus, we introduce the shape derivative to apply a gradient method for the minimization problem. Based on the previous study (Allaire et al. 2004), the shape derivative of $J$ is defined as the Fréchet derivative using the small displacement $\boldsymbol{\theta} \in$ $W^{1, \infty}\left(\mathbb{R}^{d}, \mathbb{R}^{d}\right)$, as follows:

$J^{\prime}(\Omega)(\boldsymbol{\theta})=\int_{\Gamma} v \boldsymbol{\theta} \cdot \mathbf{n} \mathrm{d} \Gamma$,

where $v$ represents a scalar function, given by

$v=j(\mathbf{u})+\boldsymbol{\epsilon}(\mathbf{u}): \mathbf{C}: \boldsymbol{\epsilon}(\tilde{\mathbf{u}})$,

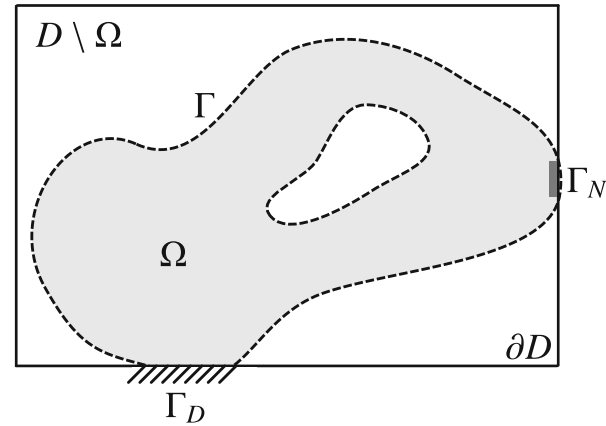

Fig. 1 Schematic diagram of working domain $D$ including the material domain $\Omega$ with boundary settings where $\tilde{\mathbf{u}} \in H^{1}(D)^{d}$ is the adjoint field that is the solution of the following adjoint problem:

$\begin{cases}-\nabla \cdot \boldsymbol{\sigma}(\tilde{\mathbf{u}})=-\left(\frac{\partial j(\mathbf{u})}{\partial \mathbf{u}}\right)^{\mathrm{T}} & \text { in } \Omega \\ \tilde{\mathbf{u}}=\mathbf{0} & \text { on } \Gamma_{D} \\ \boldsymbol{\sigma}(\tilde{\mathbf{u}}) \cdot \mathbf{n}=-\left(\frac{\partial k(\mathbf{u})}{\partial \mathbf{u}}\right)^{\mathrm{T}} & \text { on } \Gamma_{N} \\ \boldsymbol{\sigma}(\tilde{\mathbf{u}}) \cdot \mathbf{n}=\mathbf{0} & \text { on } \Gamma .\end{cases}$

In a gradient method for a shape optimization problem, a descent direction of $\boldsymbol{\theta}$ is easily revealed as $-v \mathbf{n}$.

\section{Shape representation based on the level set method}

Here, we discuss shape optimization based on the level set method, in which the boundary of shape $\Omega$ is captured based on the Eulerian approach using the iso-surface of a scalar function, the so-called level set function, during the optimization process. The novel aspect of the level set-based approach is that any part of the shape boundary is allowed to split or merge during the optimization process, whereas such topological changes are typically inhibited in classical shape optimization methods based on the Lagrangian approach.

In this study, we propose a new level set-based shape optimization method that is interpreted as an extended approach based on the previous research proposed by Allaire et al. (2004). To clarify the difference between the conventional approach and our newly proposed method, we briefly introduce the conventional approach in which the level set function is convected using an advection equation, which requires reinitialization so that the level set function retains its property as a signed distance function to ensure its smoothness during the optimization process. We then discuss our approach, in which a sinus filter is applied to the signed distance function to obtain a smooth truncation away from the structural boundaries, and the convected level set method (Ville et al. 2011) is used to avoid the need to reinitialize the level set function.

\subsection{Standard level set method}

The basic idea of the level set method is to represent a structural boundary, here, $\Gamma$, as the iso-surface of level set function $\phi: D \rightarrow \mathbb{R}$. We define the level set function $\phi$ in $D$ as follows:

$\begin{cases}\phi(\mathbf{x})=-d(\mathbf{x}, \partial \Omega) & \text { if } \mathbf{x} \in \Omega \\ \phi(\mathbf{x})=0 & \text { if } \mathbf{x} \in \partial \Omega \\ \phi(\mathbf{x})=d(\mathbf{x}, \partial \Omega) & \text { if } \mathbf{x} \in D \backslash \Omega\end{cases}$

where $\mathbf{x}$ is a point in the working domain and $d(\cdot, \partial \Omega)$ is the Euclidean distance function to $\partial \Omega$. 
The elastic problem (1) is extended to the working domain $D$ using the ersatz material approach. That is, we introduce an extended elastic tensor $\mathbf{C}^{*}$ to the elastic problem, replacing the original elastic tensor $\mathbf{C}$, as follows:

$$
\mathbf{C}^{*}=H(\phi) \mathbf{C} \text { with } H(\phi)=\left\{\begin{array}{l}
1 \text { if } \phi(\mathbf{x}) \leqslant 0 \\
\epsilon^{*} \text { if } \phi(\mathbf{x})>0,
\end{array}\right.
$$

where $\epsilon^{*}>0$ is a small value to avoid the singularity problem of the rigidity matrix, and we set $\epsilon^{*}=1.0 \times 10^{-3}$. We note that the Heaviside function $H(\phi)$ in our method here is always equal to extreme values, i.e., $H(\phi)=1$ or $\epsilon^{*}$, whereas in most previous research dealing with level setbased shape optimization problems, interpolation treatments are used, in which the Heaviside function is replaced by a continuous smoothed function.

Using the pseudo-time $t$, the boundary $\partial \Omega$ is evolved using the following advection equation:

$\frac{\partial \phi}{\partial t}+\mathbf{V} \cdot \nabla \phi=0$,

where $\mathbf{V}$ represents the convection velocity that is defined as $\mathbf{V}=-v \mathbf{n}$ in the level set-based shape optimization method (Allaire et al. 2004). Since the unit normal is given by $\mathbf{n}=$ $\nabla \phi /|\nabla \phi|,(10)$ can be reformulated as a Hamilton-Jacobi equation:

$$
\frac{\partial \phi}{\partial t}-v|\nabla \phi|=0 \text {. }
$$

An interesting feature of using the signed distance function is that we can ensure that the smoothness of the level set function is preserved, which is important when solving the advection equation with numerical schemes such as the finite element method and the finite difference method. In fact, since the property of the signed distance function, i.e., $|\nabla \phi|=1$, is not retained by just solving (10) or (11), smooth solutions are not usually guaranteed during the optimization process. To avoid this numerical instability, the level set function must be reinitialized by periodically solving the following unsteady Eikonal equation:

$\frac{\partial \phi}{\partial \tau}+\operatorname{sign}\left(\phi_{0}\right)(|\nabla \phi|-1)=0$,

where $\tau$ represents the pseudo-time for the reinitialization, and $\operatorname{sign}\left(\phi_{0}\right)$ represents the signed function with respect to the initial level set function at $\tau=0$. Equation (12) provides as a stationary solution the signed distance to the initial interface, $\phi_{0}(\mathbf{x})=0$.

One of the main limitations of a conventional level set method is that the profile of the level set function must preserve the property of a signed distance function for the computation to remain stable, whereas classical shape optimization and topology optimization methods do not require additional calculations in the form of a reinitialization scheme. In practice, with conventional level set-based approaches, this can lead to a tradeoff between substantial numerical instability or massive computational cost to achieve an optimal shape.

\subsection{Convected level set method}

To overcome the above limitation of conventional level set-based approaches, we construct a shape optimization method based on the convected level set method proposed by Ville et al. (2011). This approach was originally applied to two-phase fluid flow problems for stably capturing interfaces, and revealed that sufficient accuracy can be preserved without using a reinitialization procedure. The basic idea of the convected level set method is as follows.

Since the only information used for computation in a level set method is the zero iso-surface of the level set function, the level set function does not need to be evolved with the advection equation over the entire domain $D$. Thus, to limit treatment to only the vicinity of the structural boundary $\partial \Omega$, we introduce the following sinus filter (Coupez et al. 2015):

$\psi(\mathbf{x})=E \tanh \left(\frac{\phi(\mathbf{x})}{E}\right)$,

where $E>0$ represents a parameter for determining the thickness of the interface. As shown in Fig. 2, the profile of this filtered level set function is almost piecewise constant and smoothly truncated as $|\psi|=E$ and $|\nabla \psi| \ll 1$, when $|\phi|>2 E$. Due to the definition of the sinus filter in (13), the hyperbolic tangent function has the following property:

$E \tanh \left(\frac{\phi(\mathbf{x})}{E}\right) \rightarrow \phi(\mathbf{x})$ when $E \rightarrow \infty$.

Since $\phi(\mathbf{x})$ is the signed distance function defined in (8), our proposed method corresponds to an approach that deals with the signed distance function as $E \rightarrow \infty$.

As a simple method for capturing the structural boundary, based on the filtered level set function $\psi$, we can use the following advection equation:

$$
\frac{\partial \psi}{\partial t}-\mathbf{V} \cdot \nabla \psi=0
$$

Similarly, we can also use the Hamilton-Jacobi equation as follows:

$\frac{\partial \psi}{\partial t}-v|\nabla \psi|=0$

The key idea of using the sinus filter is that the hyperbolic tangent function has a self-determinant property, i.e., the gradient of $\psi$ satisfies the following equation:

$|\nabla \psi|=1-\left(\frac{\psi}{E}\right)^{2}:=G(\psi)$.

Thus, the unsteady Eikonal equation (12) can be easily extended as a reinitialization scheme for $\psi$ by using (17), 


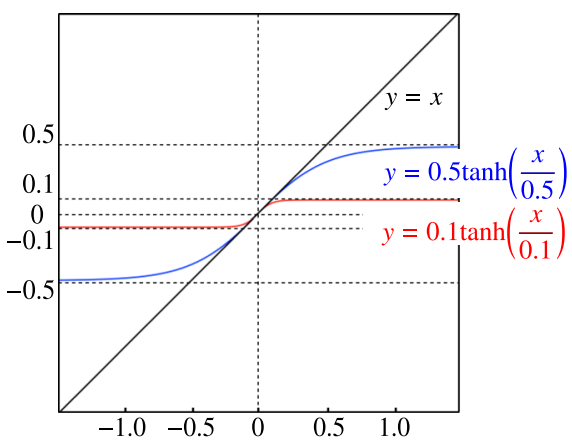

(a) Analytical functions in 1D case

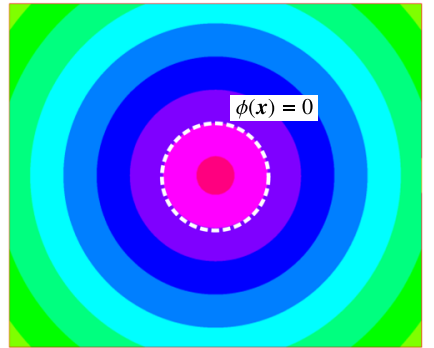

Signed distance function $\phi(\boldsymbol{x})= \pm d(\boldsymbol{x})$

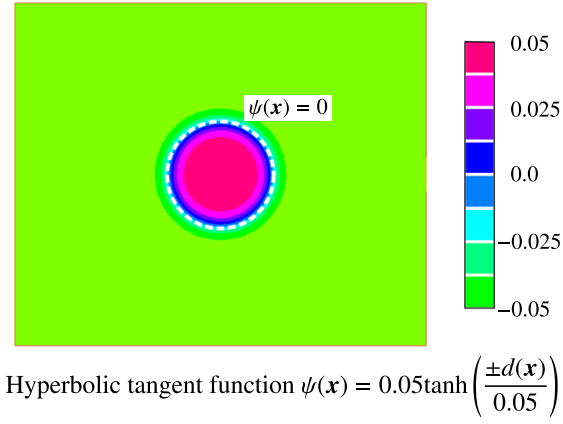

(b) Signed distance function $\phi(x)$ and hyperbolic tangent function $\psi(\boldsymbol{x})$ in 2D case

Fig. 2 Examples of hyperbolic tangent functions in one- and two-dimensional cases

instead of $|\nabla \phi|=1$ that was used for the signed distance function, as follows:

$\frac{\partial \psi}{\partial \tau}+\operatorname{sign}\left(\psi_{0}\right)(|\nabla \psi|-G(\psi))=0$,

where $\psi_{0}$ represents the initial value of $\psi$ at $\tau=0$. Several studies investigated the validity and utility of the hyperbolic tangent level set function in numerical simulations for two-phase fluid flow problems, and revealed that the computational cost for the reinitialization can be greatly reduced and the overall robustness of the numerical approach is improved (Olsson and Kreiss 2005; Olsson et al. 2007; Desjardins et al. 2008).

Although the computational cost of reinitialization can be reduced by using the sinus filter, which truncates unimportant areas in the working domain $D$, we further consider how to completely avoid the need to use additional calculations for the reinitialization treatment during the optimization process. To do this, we introduce the convected reinitialization equation proposed by Ville et al. (2011), replacing the Hamilton-Jacobi equation (16), as follows:

$\frac{\partial \psi}{\partial t}-\mathbf{V} \cdot \nabla \psi+\lambda \operatorname{sign}(\psi)(|\nabla \psi|-G(\psi))=0$,

where $\lambda=\partial \tau / \partial t$ is a parameter whose determination strategy will be described in Section 4. Since (19) contains both the interface motion and the reconstruction of $\psi$, we need only solve (19) when updating $\psi$ during the optimization process. This equation can be rewritten as

$\frac{\partial \psi}{\partial t}-(\mathbf{V}-\lambda \operatorname{sign}(\psi) \mathbf{n}) \cdot \nabla \psi=\lambda \operatorname{sign}(\psi) G(\psi)$.

We note that the reason why we use the normal gradient term in (19) and (20) is that the standard advection equation is suitable for computations when using an unstructured mesh incorporating a mesh adaptation, while the finite difference method is suitable for solving the Hamilton-Jacobi equation when using a structured mesh.

\section{Numerical implementation}

Our proposed algorithm is an iterative method based on the descent gradient method, and is structured as follows:

Step 1. The initial signed distance function $\phi(\mathbf{x}, 0)$ is set in the working domain $D$, and the initial hyperbolic tangent level set function $\psi(\mathbf{x}, 0)$ is then derived using the sinus filter in (13).

Step 2. The mesh adaptation is applied to $\psi$ to concentrate fine meshes in an area near structural boundary $\partial \Omega$, while domains beyond this area are discretized using coarse meshes.

Step 3. The elastic problem (1) based on the extended elastic tensor $\mathbf{C}^{*}$ is solved for $\mathbf{u}$ using the finite element method.

Step 4. If the value of $L$ defined in (4) is sufficiently converged, an optimal configuration is obtained and the optimization is finished, otherwise, the adjoint problem (7) is solved for $\tilde{\mathbf{u}}$ using the finite element method.

Step 5. The shape derivative in (6) is calculated using the current $\mathbf{u}$ and $\tilde{\mathbf{u}}$.

Step 6. The level set function is evolved using the convective reinitialization equation in (20), after which the optimization procedure returns to the second step of the iterative loop.

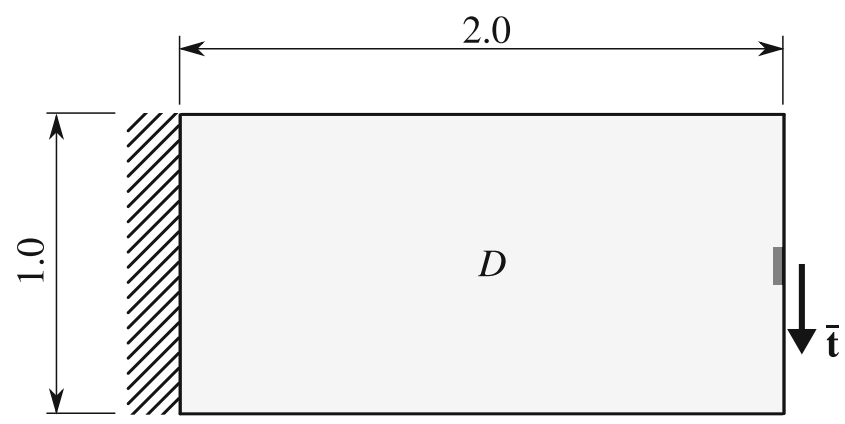

Fig. 3 Design domain and boundary conditions in cantilever problem 


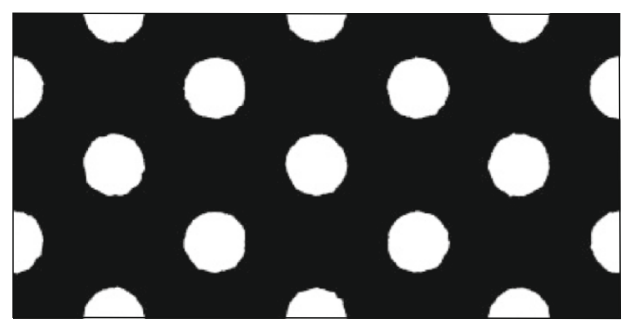

(a) Initial shape

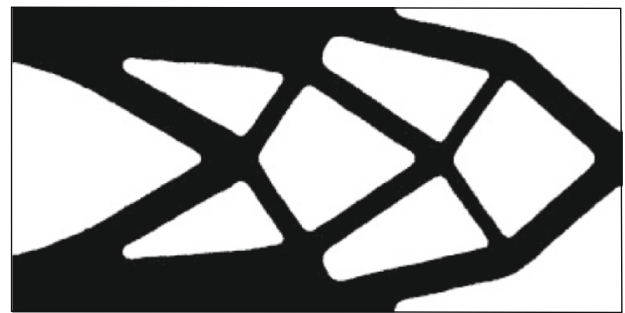

(b) Optimal shape

Fig. 4 Initial and optimal configurations in cantilever problem (black: solid; white: void)

These procedures are iterated until the following criterion for the value of $L$ is met:

$\left|\frac{L_{t}-L_{t-\Delta t}}{L_{t}}\right|<\epsilon_{\mathrm{opt}}$,

where subscript $t$ represents the number of iterations carried out during the optimization process and $\Delta t$ is a single iteration. We set this criterion so that $\epsilon_{\mathrm{opt}}=1.0 \times 10^{-4}$.

Our proposed method employs an anisotropic unstructured mesh adaptation scheme (Castro-Diaz et al. 1997), in order to increase the computational accuracy of the time evolution equation for the hyperbolic tangent level set function. The basic idea of the mesh adaptation is that fine meshes are generated based on the gradient information of the target variable, defined as $\psi$ in this study. As mentioned previously, since $|\nabla \psi| \ll 1$ is satisfied almost everywhere in $D$ except in the vicinity of $\partial \Omega$, appropriate fine meshes can be automatically generated near the structural boundary. Since $\psi$ is smoothly truncated due to the definition of the hyperbolic tangent function in (13), the mesh gradually becomes coarser in the non-gradient domain as distance increases from the vicinity of $\partial \Omega$. Furthermore, the thickness of this transition phase between the finest mesh and the coarsest mesh is uniformly distributed without any treatment, owing to the property of the hyperbolic tangent function in (17). We therefore note that the hyperbolic tangent level set function and the mesh adaptation are highly compatible.

The time evolution equation for $\psi$ in (20) is discretized as $\mathbb{P}^{1}$ Lagrange finite elements in $D$, in which the state and adjoint fields are also discretized using $\mathbb{P}^{1}$ elements. Since the convective reinitialization equation is nonlinear, the nonlinear terms in (20) are linearized by evaluating the value of $\psi$ at the previous time step. We note that (20) need not be solved exactly, but $\psi$ should retain its smoothness and locality as far possible. Since unstructured meshes are used in the treatment of the linearized convective reinitialization equation in our method, the linearized equation is solved using the method of characteristics, which is especially suitable when using unstructured meshes. Details of the operation of this method are available elsewhere (Pironneau 1982). Our method is based on an approach (Strain 1999) that has applied the method of characteristics to a level set method. The basic idea of this method and its application to solve

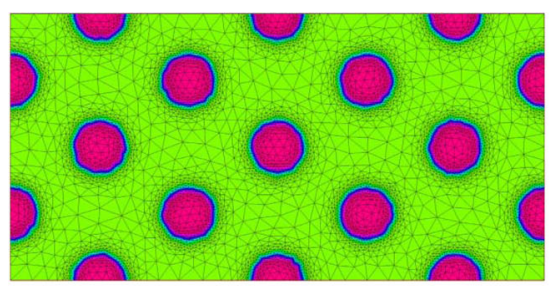

(a) Initial shape

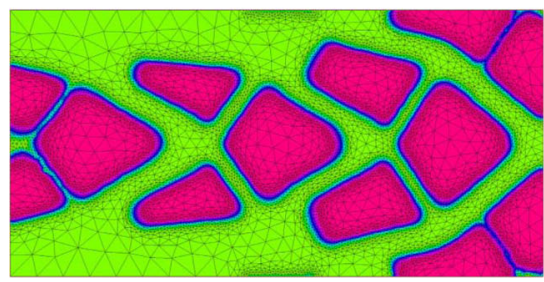

(d) Step 20

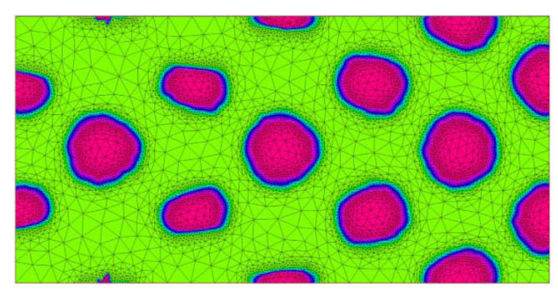

(b) Step 5

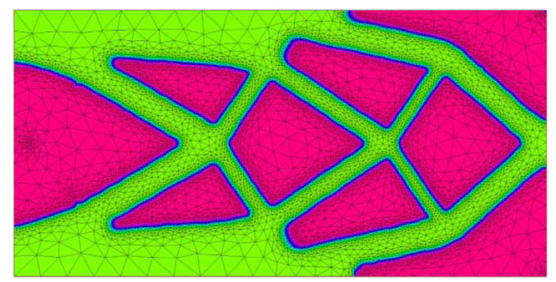

(e) Step 50

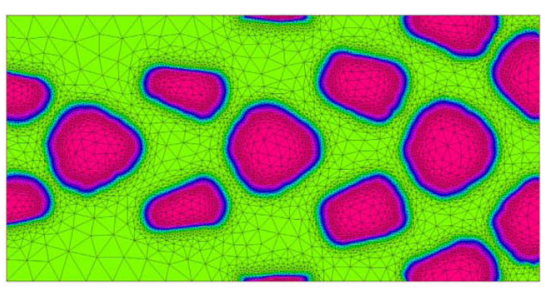

(c) Step 10

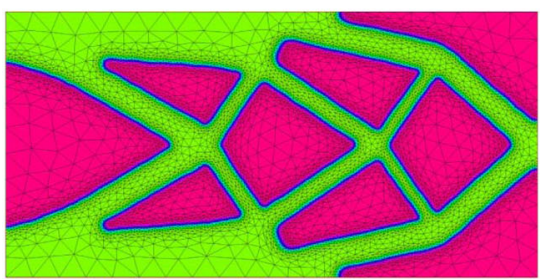

(f) Optimal shape

Fig. 5 Distribution of the hyperbolic tangent level set function $\psi$ in working domain $D$ during optimization process in mean compliance minimization problem 


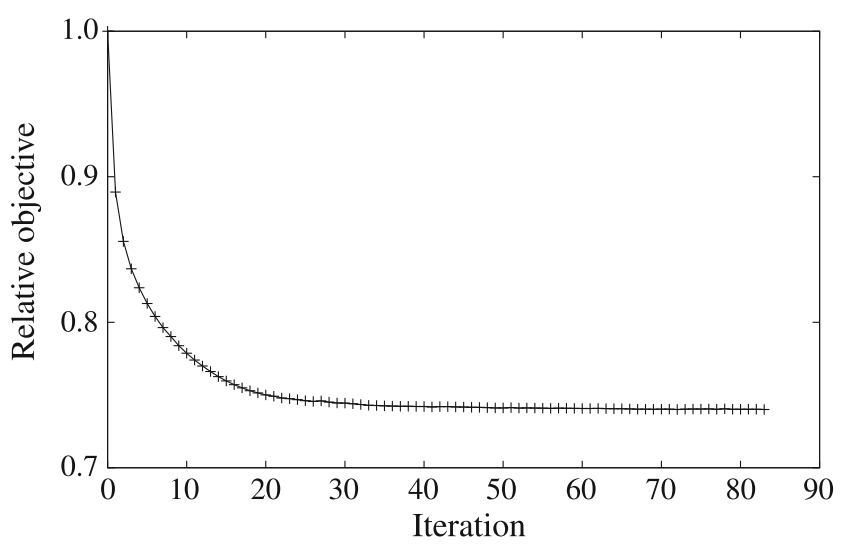

Fig. 6 History of relative objective functional in the mean compliance minimization problem. The objective functional value is normalized to its initial value

(20) are shown in Appendix A. To ensure stable computation, $\lambda$ in (20) is chosen so that $\lambda \sim h_{\min } / \Delta t$, where $h_{\text {min }}$ and $\Delta t$ are the minimal element edge size and the time step, respectively (Ville et al. 2011).

Although the shape derivative in (6) only exists on the structural boundary $\partial \Omega$, the scalar field $v$ should nevertheless be defined in $D$ or a vicinity of $\partial \Omega$. This is a necessary requirement for the numerical setting of the level set method. In addition, since $v$ is typically dependent on the derivatives of the solution u, e.g., $v \in L^{2}(\Gamma)$, it is obvious that the smoothness of $v$ is not guaranteed during the optimization process. Since this feature can often causes numerical instability, it is numerically advantageous to regularize the scalar field $v$ to provide some degree of smoothness. One way to extend and regularize the scalar field $v$ is to solve the variational formulation for $v^{\text {reg }} \in$ $H^{1}(D)$, as follows (Allaire et al. 2014):

$\int_{D}\left(v^{\mathrm{reg}} z+\alpha \nabla v^{\mathrm{reg}} \cdot \nabla z\right) \mathrm{d} \Omega=J^{\prime}(\Omega)(z \mathbf{n})$ for $\forall z \in H^{1}(D)$, where $\alpha>0$ is a small parameter that determines the regularization width, and this variation formulation represents a mapping of the shape derivative to $H^{1}(D)$ from the original space, e.g., $L^{2}(\Gamma)$. The parameter $\alpha$ is typically of the order of the minimal element edge size $h_{\min }$, and is set to the value of $10 h_{\min }^{2}$ in this study. We note that the regularized scalar field $v^{\text {reg }}$ does not lose its property as a shape derivative when the direction of descent for $J$ is given by $-v \mathbf{n}$. That is, choosing $z=-v^{\text {reg }}$, we find that

$J^{\prime}(\Omega)\left(-v^{\text {reg }} \mathbf{n}\right)=-\int_{D}\left(\left(v^{\text {reg }}\right)^{2}+\alpha\left|\nabla v^{\text {reg }}\right|^{2}\right) \mathrm{d} \Omega \leqslant 0$,

which shows that $-v^{\text {reg }} \mathbf{n}$ guarantees a descent direction for $J$.

In this research, all numerical computations were performed using FreeFem++ (Hecht 2012).

\section{Numerical examples}

Here we provide two numerical examples to confirm the validity of the proposed method. The normalized Young's modulus and Poisson's ratio were set as 1.0 and 0.3 , respectively.

\subsection{Mean compliance minimization problem}

Our first example is applied to a stiffness maximization problem for a two-dimensional cantilever, as illustrated in Fig. 3. The computational domain $D$ is a $2.0 \times 1.0$ rectangle with a fixed boundary condition on the left side and a traction boundary condition on a small area near the center of the right-hand side. The domain $D$ is discretized using an unstructured mesh whose minimum and maximum element edge sizes are set so that $h_{\min }=0.01$ and $h_{\max }=0.1$,
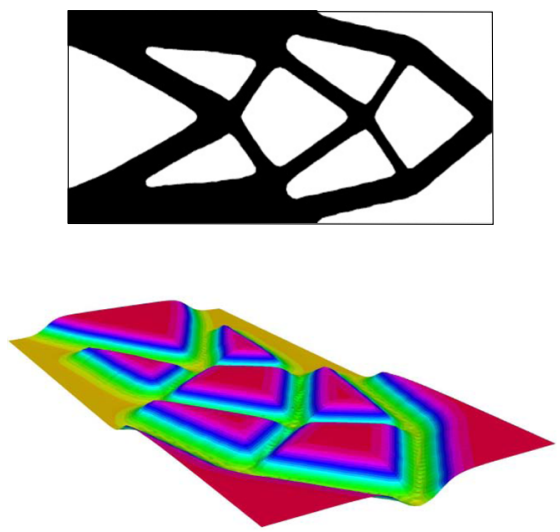

(a) $E=h_{\min }$
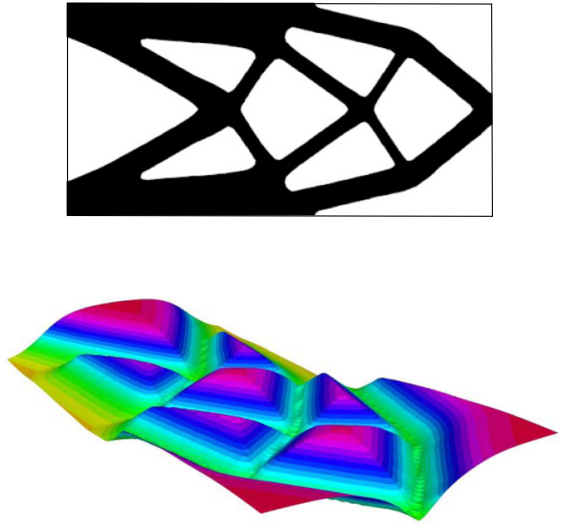

(b) $E=3 h_{\min }$
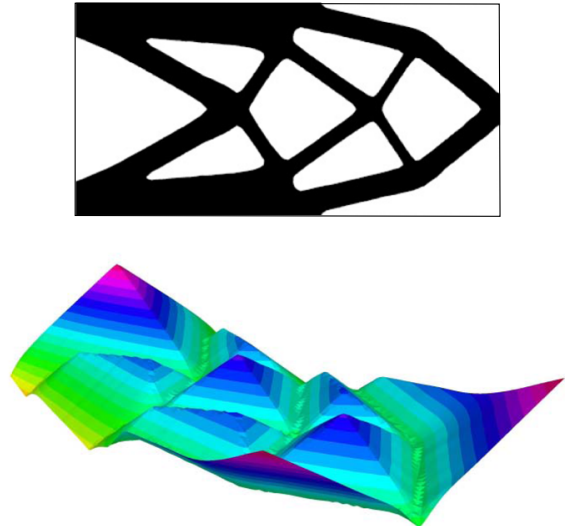

(c) Signed distance function

Fig. 7 Optimal results for different settings of $E:(\mathbf{a}) E=\bar{h}_{\min }$; (b) $E=3 \bar{h}_{\min }$; (c) Signed distance function (corresponding to $E \rightarrow \infty$ ) 


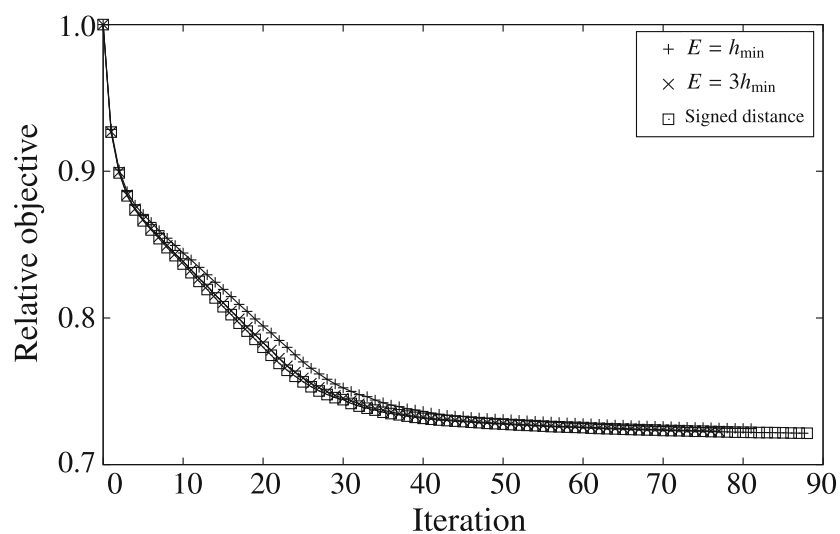

Fig. 8 History of relative objective functional for three cases: $E=$ $h_{\mathrm{min}} ; E=3 h_{\mathrm{min}}$; Signed distance function (corresponding to $E \rightarrow$ $\infty)$

respectively. The time step and the parameter $\lambda$ were set to $\Delta t=0.03$ and $\lambda=0.1$, respectively. The regularization coefficient was set as $l=1.0$.

For structural optimization in a stiffness maximization problem, the objective functional $J_{1}(\Omega)$ is typically defined as the mean compliance, as follows:

$J_{1}(\Omega)=\int_{\Gamma_{N}} \overline{\mathbf{t}} \cdot \mathbf{u} \mathrm{d} \Gamma$.

In this case, it is well-known that the mean compliance minimization problem is self-adjoint, i.e., $\tilde{\mathbf{u}}=-\mathbf{u}$. Thus, we do not need to solve the adjoint problem (7).

Figure 4 shows the initial and optimal shapes, and the close similarity to the optimal result obtained by Allaire et al. (2004) confirms that our method obtains an appropriate result. The distribution of the hyperbolic tangent level set function $\psi$ together with the mesh in $D$ is shown in Fig. 5, and we can confirm that the mesh adaptation works appropriately in the vicinity of the structural boundary. The convergence history of $J_{1}(\Omega)$ is shown in Fig. 6, and the value of $J_{1}(\Omega)$ is monotonically converged by approximately the 50th step. We emphasize that since our proposed

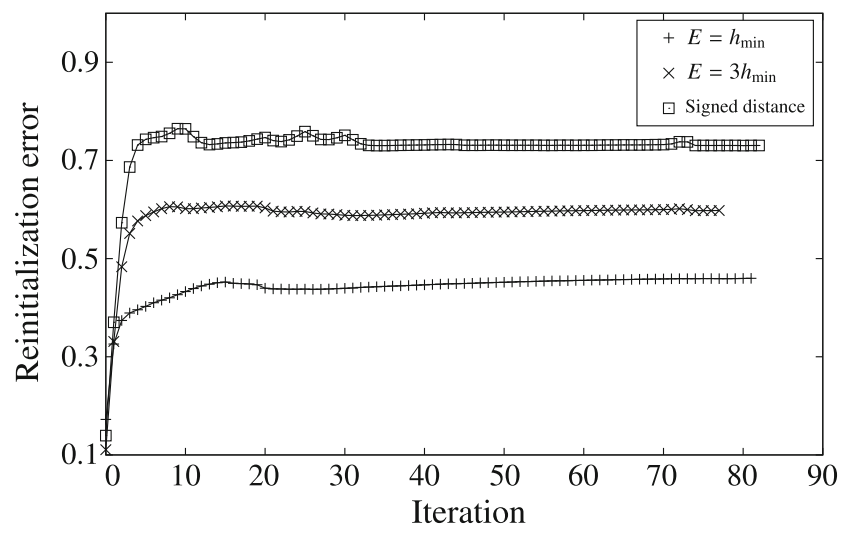

Fig. 9 History of reinitialization error norm $\epsilon_{\psi}$ in three cases: $E=$ $\bar{h}_{\min } ; E=3 \bar{h}_{\mathrm{min}}$; signed distance function

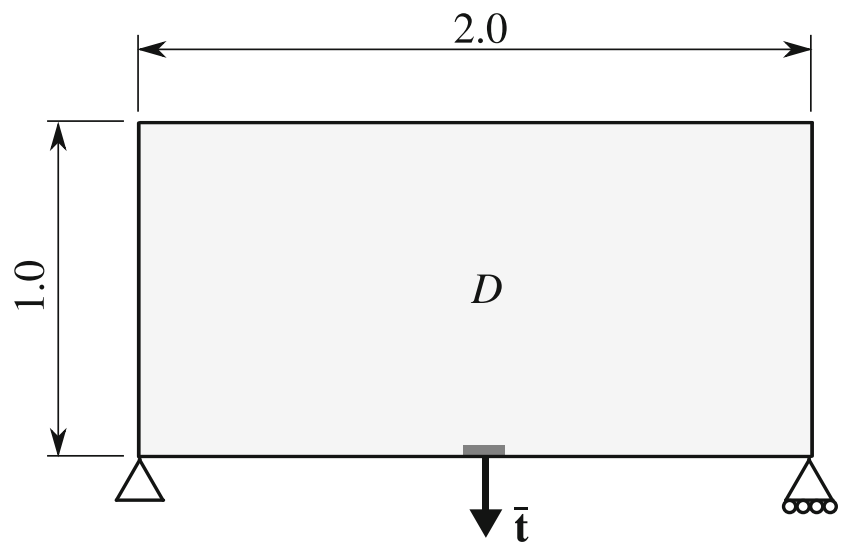

Fig. 10 Design domain and boundary conditions in bridge problem

method is based on the convected level set method, the additional reinitialization calculation is completely avoided during the optimization process.

Next, the effect of parameter $E$ settings on optimal configurations was investigated in a cantilever problem. Here, we introduced an error norm $\epsilon_{\psi}$ to evaluate the reinitialization error of $\psi$, defined as

$\epsilon_{\psi}=\||\nabla \psi(\mathbf{x})|-G(\psi)\|_{L^{2}(D)}$.

For an equal evaluation with respect to the error norm under the same degrees of freedom, we used a structured mesh of square elements whose edge size is set to $\bar{h}_{\text {min }}=0.02$, without the mesh adaptation and convective reinitialization. Additionally, the Hamilton-Jacobi equation in (16) was used
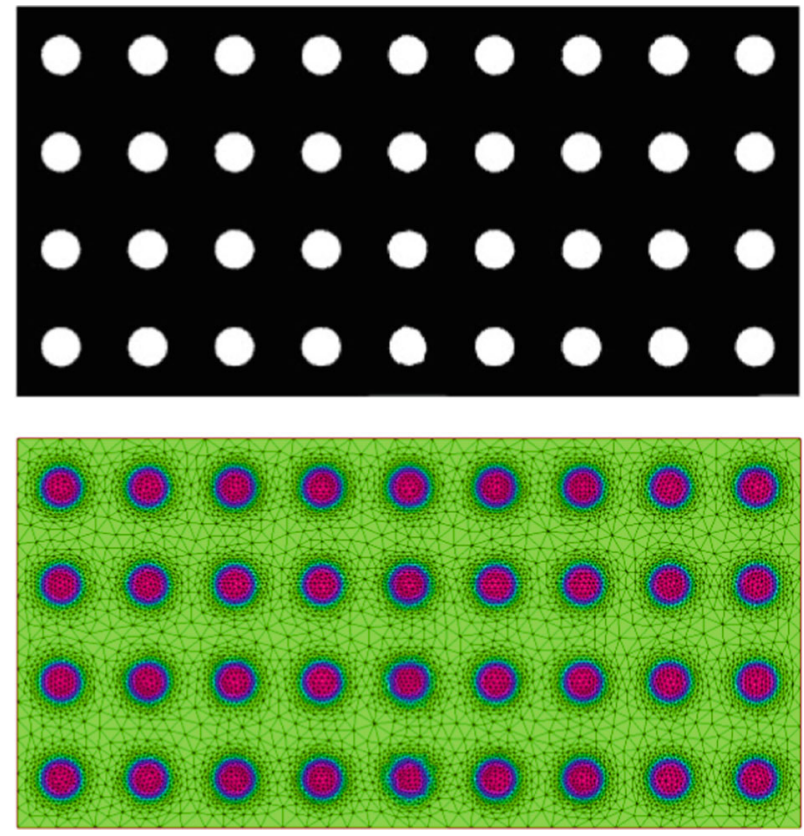

Fig. 11 Initial configuration (top) and initial distribution of the hyperbolic tangent level set function $\psi$ (bottom) 

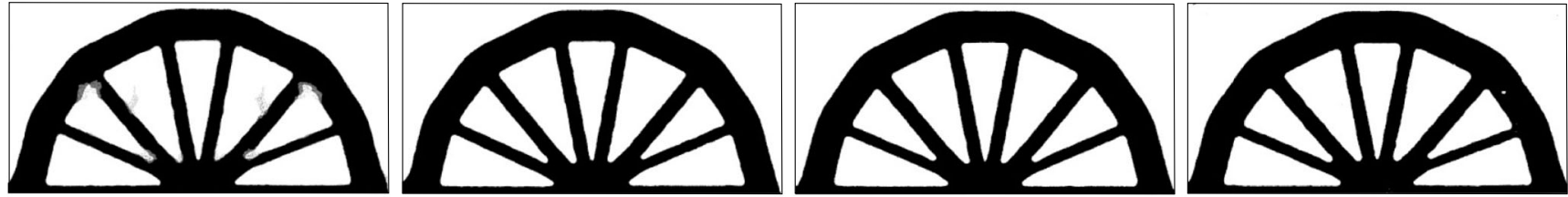

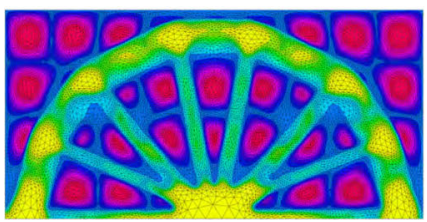

(a) $\lambda=0.0$

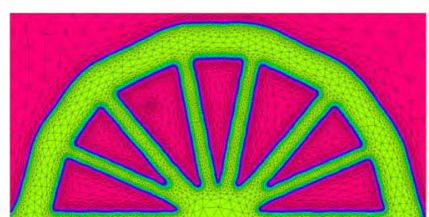

(b) $\lambda=0.01$

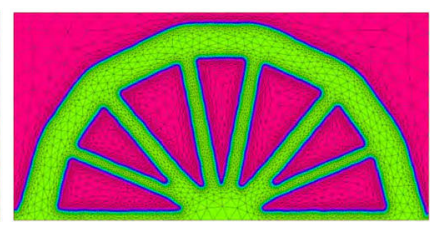

(c) $\lambda=0.1$

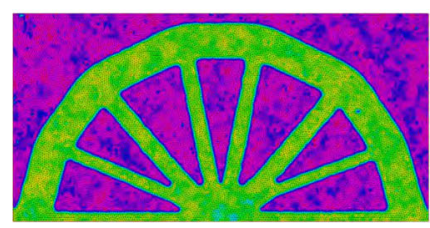

(d) $\lambda=0.4$

Fig. 12 Optimal results in different settings of $\lambda$, where (a) $\lambda=0.0$; (b) $\lambda=0.01$; (c) $\lambda=0.1$; (d) $\lambda=0.4$

for evolving the hyperbolic tangent level set function $\psi$, and the unsteady Eikonel equation in (18) was used for the reinitialization. Based on the previous approach proposed by (Allaire et al. 2004), the finite difference scheme was employed for solving (16) and (18), in which the unsteady Eikonel equation in (18) was iteratively calculated 10 times during each optimization step.

Figure 7 shows the optimal results for the cantilever problem for different set values of $E$ : (a) $E=\bar{h}_{\text {min }}$; (b) $E=3 \bar{h}_{\min }$; and (c) signed distance function (corresponding to $E=\infty$ ). The convergence histories of the objective functional for three different settings of $E$ are shown in Fig. 8, and we can confirm that these convergence behaviors are almost identical. Figure 9 shows the history of error norm $\epsilon_{\psi}$ during the optimization process for the different settings of $E$, corresponding to the optimal shapes in Fig. 7. As shown in Fig. 9, the reinitialization error becomes larger as $E$ is set to larger values. This is because setting $E$ to a small value restricts the reinitialization error, since $|\nabla \psi| \ll 1$ almost everywhere in $D$ except in the vicinity of $\psi=0$. Due to the relationship between $\psi$ and $\phi$ in (14), this behavior of $E$ indicates that the use of hyperbolic tangent function

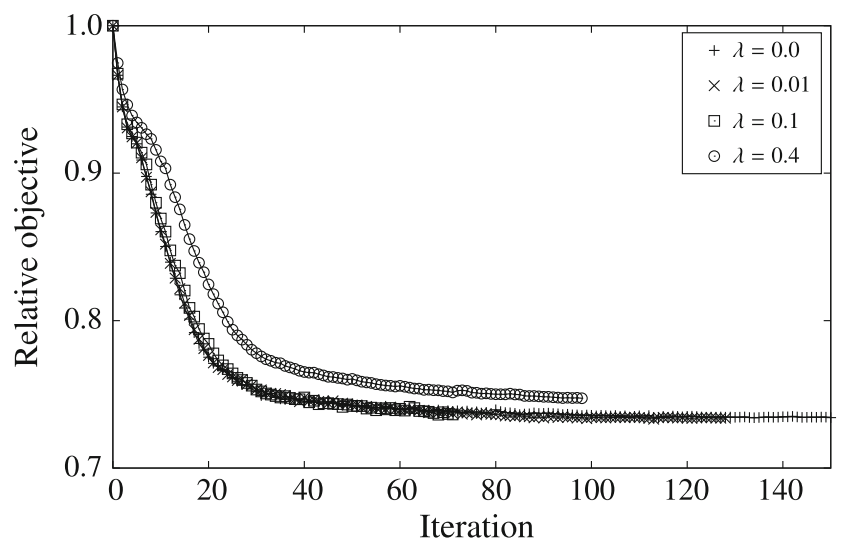

Fig. 13 History of relative objective functional for four cases: $\lambda=$ $0.0 ; \lambda=0.01 ; \lambda=0.1 ; \lambda=0.4$ reduces the computational cost for the reinitialization, compared with the use of the signed distance function. Thus, when using the convected level set method, $E$ should be set as small as possible so that the process of updating $\psi$ will be stable. According to our numerical tests, $E=h_{\min }$ was a good choice and provided accurate reinitialization in all numerical examples that we treated in this paper.

As mentioned in the previous section, $\lambda$ must be chosen so that $\lambda \sim h_{\min } / \Delta t$. To reveal the appropriate value of $\lambda$, we investigated the dependency of the optimal shape with respect to different settings for $\lambda$. Here, we consider the stiffness maximization problem illustrated in Fig. 10, where the domain $D$ was discretized using unstructured meshes whose minimum and maximum element edge sizes were set so that $h_{\min }=0.01$ and $h_{\max }=0.1$, respectively, with the mesh adaptation applied. In this optimization problem, we used the same initial design, shown in Fig. 11, that is used in all cases for the optimization problem shown in Fig. 10.

Figure 12 shows the optimal results in which different values of $\lambda$ were set as follows: (a) 0.0 ; (b) 0.01 ; (c) 0.1 ; and (d) 0.4 . We can confirm that the optimal results shown in Figs. $12 \mathrm{~b}$ and $\mathrm{c}$ are practically identical.

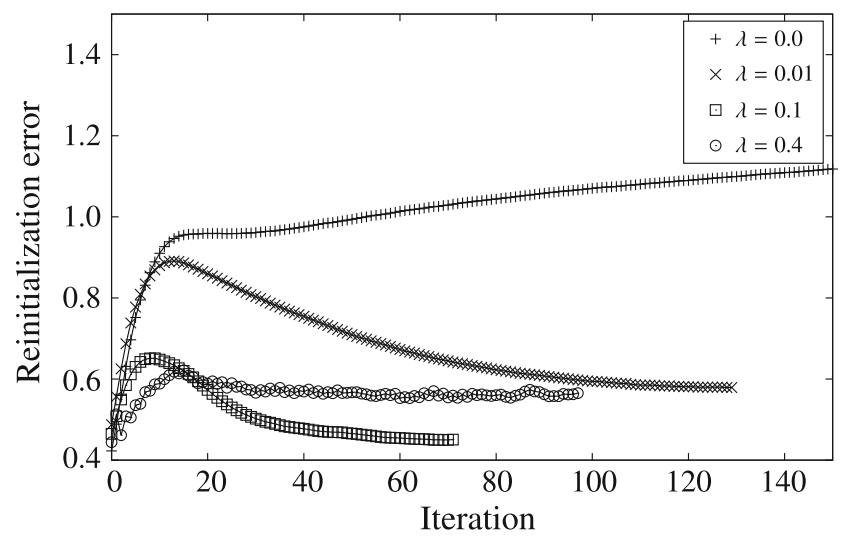

Fig. 14 History of reinitialization norm $\epsilon_{\psi}$ for four cases: $\lambda=0.0$; $\lambda=0.01 ; \lambda=0.1 ; \lambda=0.4$ 


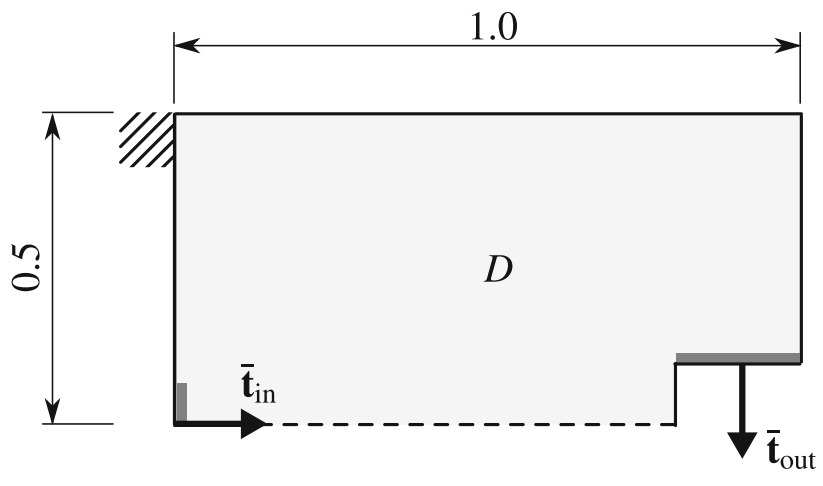

Fig. 15 Design domain and boundary conditions in design of compliant mechanism

On the other hand, the optimal result shown in Fig. 12a is the outcome without reinitialization, which means that only the Hamilton-Jacobi equation in (16) was solved during the optimization process. In this case, the distribution of the level set function $\psi$ does not have the property of the hyperbolic tangent function, and the optimal result is therefore affected by the numerical instability that occurs due to the lack of reinitialization treatment. Although the optimal result shown in Fig. 12d may be an appropriate result, the distribution of $\psi$ was unstable. In fact, the optimization calculation diverged when $\lambda \geqslant 0.5$. The convergence histories of the objective functional for four different settings of $\lambda$ are shown in Fig. 13, and we can confirm that these convergence behaviors are almost identical, except the case where $\lambda=0.4$.

Figure 14 shows the histories of the error norm $\epsilon_{\psi}$ for different settings of $\lambda$ corresponding to the results shown in Fig. 12. We note that $\lambda=0.1$ was a good choice not only for this problem, but also for all the numerical examples in this paper, as it provided stability in the convective reinitialization computations.

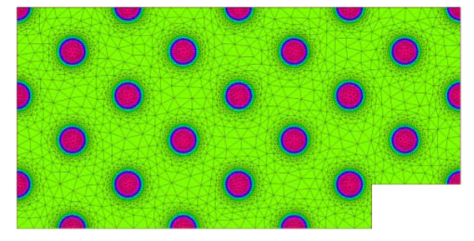

Initial shape

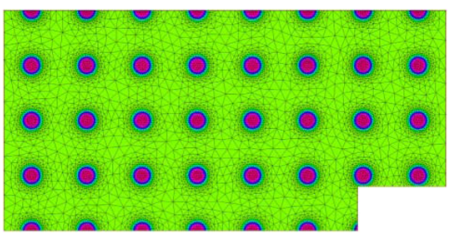

Initial shape

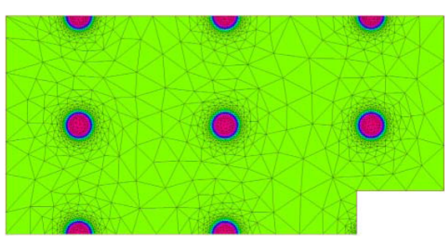

Initial shape

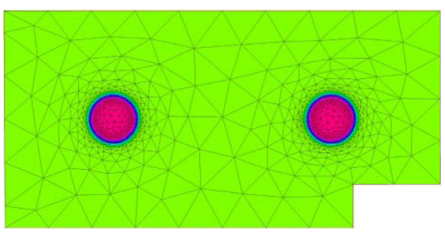

Initial shape

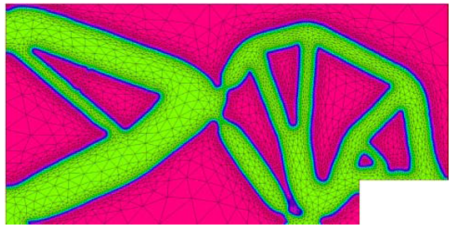

Optimal shape

(a) Case 1

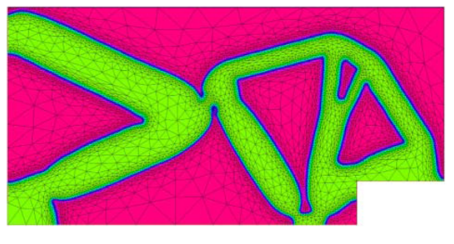

Optimal shape

(b) Case 2

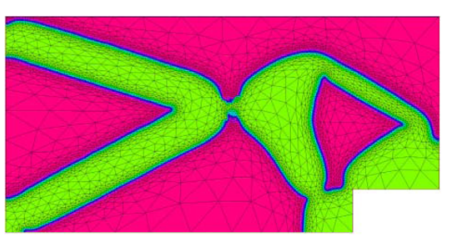

Optimal shape

(c) Case 3

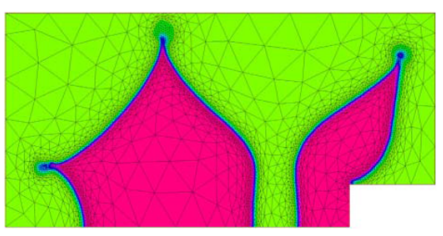

Optimal shape

(d) Case 4

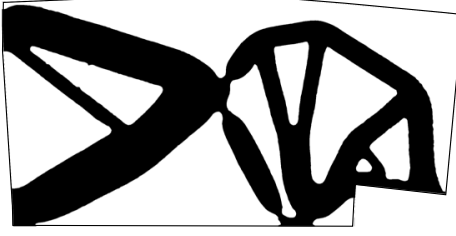

Deformed shape

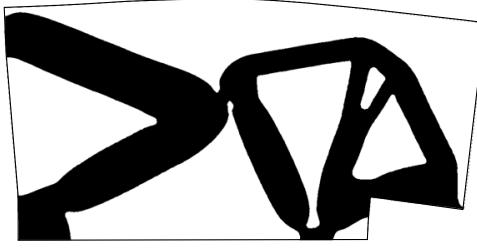

Deformed shape

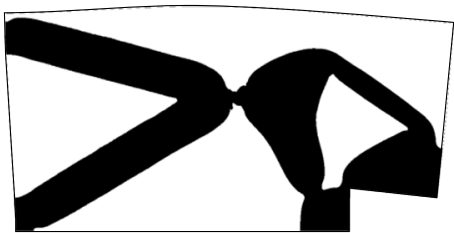

Deformed shape

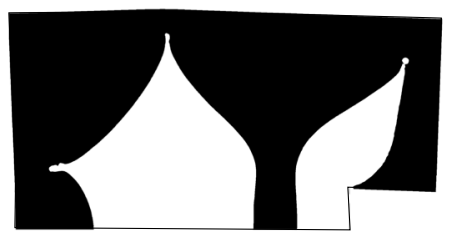

Deformed shape

Fig. 16 Four different Initial and optimal shapes in design of compliant mechanism, showing the deformed shape of each optimal shape 


\subsection{Design of compliant mechanism}

Our second example is a compliant mechanism design problem, as illustrated in Fig. 15, where the traction boundary is set so that traction $\overline{\mathbf{t}}_{\text {in }}$ is imposed at $\Gamma_{\text {in }}$. The domain $D$ is discretized using an unstructured mesh whose minimum and maximum element edge sizes were set so that $h_{\min }=0.005$ and $h_{\max }=0.1$, respectively. The time step and the parameter $\lambda$ were set to $\Delta t=0.005$ and $\lambda=0.1$, respectively. The regularization coefficient was set as $l=1.0$.

Based on the seminal work proposed by Sigmund (1997), the objective functional $J_{2}(\Omega)$ for the design of compliant mechanism is defined as

$J_{2}(\Omega)=-\int_{\Gamma_{\text {out }}} \overline{\mathbf{t}}_{\text {out }} \cdot \mathbf{u} \mathrm{d} \Gamma$,

where $\overline{\mathbf{t}}_{\text {out }}$ is a dummy traction vector representing the direction of the specified deformation at output boundary $\Gamma_{\text {out }}$. In this approach, a spring is located at the output boundary $\Gamma_{\text {out }}$, and sufficient stiffness of the material domain $\Omega$ is obtained by maximizing the mutual mean compliance (Nishiwaki et al. 1998; Nishiwaki et al. 2001). That is, the specified deformation is maximized under the condition that a sufficient stiffness is preserved. We note that since this design problem is not self-adjoint, the adjoint problem based on (7) must be solved.

We investigated the dependency of the optimal shape with respect to the initial design. The initial and optimal shapes are shown in Fig. 16, and we can confirm that all examples successfully achieved optimal shapes that can deform in a specified direction. The convergence histories of the objective functional for four cases in the compliant mechanism design problem are shown in Fig. 17. The numerical instability often observed when an optimal design has a hinge structure is evident (Fig. 16). In previous research (Allaire et al. 2004) employing a line search technique, the time step $\Delta t$ for solving the HamiltonJacobi equation is adjusted so that $L_{t} \leqslant L_{t-\Delta t}$ is satisfied

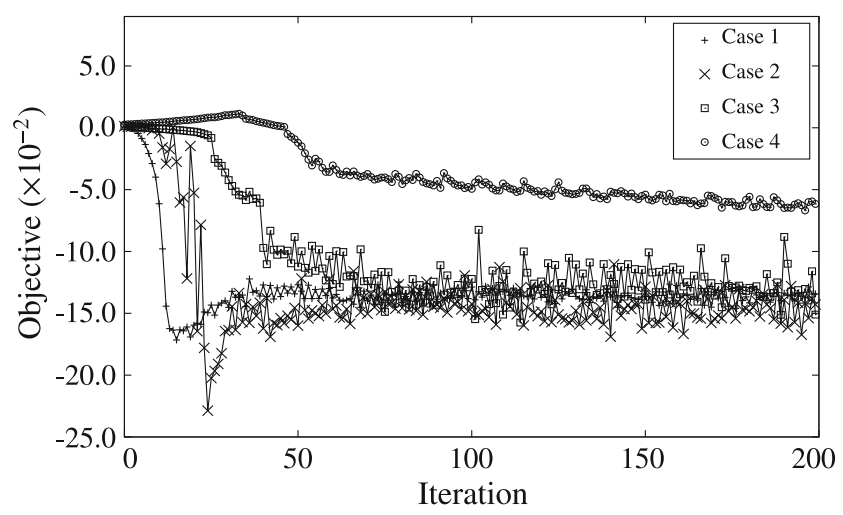

Fig. 17 History of objective functional for four cases in Fig. 16 during the optimization process. The numerical instability we encountered can be avoided by using this kind of special treatment, but this incurs additional calculations to determine an appropriate value of $\Delta t$, and significant slowing of convergence. For these reasons, and for simplicity, the optimization was carried out until the 200th step in this study.

Since the proposed method is based on the concept of shape optimization, in which the optimal shape is obtained by moving the structural boundary, the resulting optimal shape is strongly dependent on the initial design. This characteristic of level set-based shape optimization was also confirmed in previous research (Allaire et al. 2004). To overcome this problem, Allaire et al. (2005) proposed a methodology incorporating the idea of the bubble method (Eschenauer et al. 1994), which enables local minima to be avoided and enables feasible optimal results to be obtained for the most part. We note that although we do not address the local minima issue in this paper, our proposed method can be naturally expanded to a method incorporating the bubble method.

\section{Conclusion}

This paper proposed a new shape optimization method based on the convected level set method. The presented approach was applied to standard elastic problems. We achieved the following:

(1) The optimization problem for the linearized elastic structure was formulated based on the level set method in which a sinus filter was applied to map the original signed distance function to a smooth function defined as the hyperbolic tangent function. In addition, convective reinitialization was applied to the level set function to avoid the the need for a reinitialization procedure.

(2) An optimization algorithm was constructed based on the formulation of our proposed method. The analysis domain was discretized based on the finite element method in which an unstructured mesh was used. A time evolution equation based on the convected level set method was solved using the method of characteristics, and a mesh adaptation was utilized to concentrate fine meshes in the vicinity of the structural boundary so that the convected reinitialization equation could be precisely solved.

(3) Numerical examples for two-dimensional elastic problems were provided to confirm the validity and utility of the proposed method for the mean compliance minimization problem and a design problem for a compliant mechanism. The proposed method 
provided optimal results for the mean compliance minimization problem similar to those obtained in previous research. By introducing an error norm with respect to the specified profile of the level set function, we confirmed that the hyperbolic tangent profile is stably retained during the optimization process. Furthermore, optimal results were obtained for the compliant mechanism design problem and we investigated the dependency of the optimal shapes with respect to the initial designs. Based on our results, we confirmed that the obtained optimal configurations are strongly dependent on the initial design, but lowering the criteria was achieved for all examples.

Acknowledgments The authors would like to express their gratitude to Prof. Frédéric Hecht, Prof. François Jouve, and Prof. Thierry Coupez for their helpful discussion of this work. The first author was able to conduct overseas research in France supported by JSPS KAKENHI, Grant-in-Aid for JSPS Fellows, 14J02008.

\section{Appendix A: Update scheme for the level set function based on the method of characteristics}

Here, we discuss the basic idea of the method of characteristics and its application to solve the time evolution equation in (20).

For this brief explanation, we first deal with the simple advection equation (15). Consider a vector field $\mathbf{X}$ that satisfies the following ordinary differential equation,

$\frac{\partial \mathbf{X}(t)}{\partial t}=\mathbf{V}(\mathbf{X}(t), t)$

The time derivative of the composite function $\psi \circ \mathbf{X}(t)=$ $\psi(\mathbf{X}(t), t)$ is obtained as follows:

$$
\begin{aligned}
\frac{\mathrm{d} \psi(\mathbf{X}(t), t)}{\mathrm{d} t} & =\frac{\partial \psi(\mathbf{X}(t), t)}{\partial t}+\mathbf{V} \cdot \nabla \psi(\mathbf{X}(t), t) \\
& =\frac{\mathrm{D} \psi(\mathbf{X}(t), t)}{\mathrm{D} t},
\end{aligned}
$$

where $\mathrm{D} \psi / \mathrm{D} t$ represents the material derivative of $\psi$ and corresponds to the left-hand side of (15). Based on the backward Euler scheme and using a time step $\Delta t$, this can be discretized as

$$
\begin{aligned}
\frac{\mathrm{D} \psi(\mathbf{X}(t), t)}{\mathrm{D} t}= & \frac{\psi(\mathbf{X}(t), t)-\psi(\mathbf{X}(t-\Delta t), t-\Delta t)}{\Delta t} \\
& +O(\Delta t) .
\end{aligned}
$$

We now assume that the discrete time is defined as $0<t_{1}<$ $\cdots<t_{n}<\cdots<t_{N}$ with $t_{n}=n \Delta t$, and consider the following initial value problem:

$$
\left\{\begin{array}{l}
\frac{\partial \mathbf{X}(t)}{\partial t}=\mathbf{V}(\mathbf{X}(t), t) \text { for } t \in\left(t_{n-1}, t_{n}\right) \\
\mathbf{X}\left(t_{n}\right)=\mathbf{x}
\end{array}\right.
$$

Using an approximate solution, $\mathbf{X}^{n}(\mathbf{x}):=\mathbf{x}-\mathbf{V}\left(\mathbf{x}, t_{n}\right) \Delta t$, we obtain the following relation:

$\mathbf{X}\left(t_{n-1}\right)-\mathbf{X}^{n}(\mathbf{x})=O\left(\Delta t^{2}\right)$.

Consequently, (A.3) can be reformulated as

$\frac{\mathrm{D} \psi\left(\mathbf{x}, t_{n}\right)}{\mathrm{D} t}=\frac{\psi\left(\mathbf{x}, t_{n}\right)-\psi\left(\mathbf{X}^{n}(\mathbf{x}), t_{n-1}\right)}{\Delta t}+O(\Delta t)$

Using a test function $\tilde{\psi}$, the notation $\psi^{n}:=\psi\left(\cdot, t_{n}\right)$, and the scalar product $(\cdot, \cdot)$ in $L^{2}(D)$, the material derivative of $\psi^{n}$ can be approximated as follows:

$\left(\frac{\mathrm{D} \psi^{n}}{\mathrm{D} t}, \tilde{\psi}\right) \approx\left(\frac{\psi^{n}-\psi^{n-1} \circ \mathbf{X}^{n}}{\Delta t}, \tilde{\psi}\right)$,

where $\psi$ and $\tilde{\psi}$ are chosen from a finite element space. Due to the definition of $\mathbf{V}=v \mathbf{n}$ with $\mathbf{n}=\nabla \psi /|\nabla \psi|$, it should be noted that we need to assume that $\mathbf{V}\left(\mathbf{x}, t_{n}\right) \approx \mathbf{V}\left(\psi^{n-1}\right)$, to avoid the nonlinearity in (A.7).

Equation (A.7) can be only used for solving (15), so it must be expanded in order to solve (20) in which the convection velocity is defined as $(\mathbf{V}-\lambda \operatorname{sign}(\psi) \mathbf{n})$ instead of only $\mathbf{V}$ as it is in (15). Considering the source term $\lambda \operatorname{sign}(\psi) G(\psi)$, (20) can be approximated as

$$
\begin{aligned}
& \left(\frac{\mathrm{D} \psi^{n}}{\mathrm{D} t}-\lambda \operatorname{sign}\left(\psi^{n}\right) G\left(\psi^{n}\right), \tilde{\psi}\right) \\
& \approx\left(\frac{\psi^{n}-\psi^{n-1} \circ \mathbf{X}^{n}}{\Delta t}-\lambda \operatorname{sign}\left(\psi^{n-1}\right) G\left(\psi^{n-1}\right), \tilde{\psi}\right),
\end{aligned}
$$

where $\mathbf{X}^{n}=\mathbf{x}-\left(\mathbf{V}\left(\mathbf{x}, t_{n}\right)-\lambda \operatorname{sign}\left(\psi^{n-1}\right) \mathbf{n}\right) \Delta t$. Note that the use of $\operatorname{sign}\left(\psi^{n-1}\right)$ and $G\left(\psi^{n-1}\right)$ in the right-hand side of (A.8) is necessary to avoid the nonlinearity.

\section{References}

Allaire G, Jouve F (2008) Minimum stress optimal design with the level set method. Engrg Anal Bound Elem 32(11):909-918

Allaire G, Jouve F, Toader A (2004) Structural optimization using sensitivity analysis and a level-set method. J Comput Phys 194(1):363-393

Allaire G, De Gournay F, Jouve F, Toader A (2005) Structural optimization using topological and shape sensitivity via a level set method. Control Cybern 34(1):59-80

Allaire G, Dapogny C, Frey P (2014) Shape optimization with a level set based mesh evolution method. Comput Methods Appl Mech Eng 282:22-53

Allen SM, Cahn JW (1979) A microscopic theory for antiphase boundary motion and its application to antiphase domain coarsening. Acta Metall 27(6):1085-1095

Amstutz S, Andrä H (2006) A new algorithm for topology optimization using a level-set method. J Comput Phys 216(2):573-588

Bendsøe MP KikuchiN (1988) Generating optimal topologies in structural design using a homogenization method. Comput Methods Appl Mech Eng 71(2):197-224

Cahn JW, Hilliard JE (1958) Free energy of a nonuniform system. I. Interfacial free energy. Chin J Chem Phys 28(2):258-267 
Castro-Diaz M, Hecht F, Mohammadi B, Pironneau O (1997) Anisotropic unstructured mesh adaption for flow simulations. Int J Numer Methods Fluids 25(4):475-491

Challis VJ, Guest JK (2009) Level set topology optimization of fluids in Stokes flow. Int J Numer Methods Engng 79(10):12841308

Coupez T, Silva L, Hachem E (2015) Implicit boundary and adaptive anisotropic meshing. In: Perotto S, Formaggia L (eds) New challenges in grid generation and adaptivity for scientific computing, SEMA SIMAI springer series, vol 5, springer international publishing, pp 1-18

Desjardins O, Moureau V, Pitsch H (2008) An accurate conservative level set/ghost fluid method for simulating turbulent atomization. J Comput Phys 227(18):8395-8416

Duan XB, Ma YC, Zhang R (2008) Shape-topology optimization for Navier-Stokes problem using variational level set method. J Comput Appl Math 222(2):487-499

Eschenauer HA, Kobelev VV, Schumacher A (1994) Bubble method for topology and shape optimization of structures. Struct Optimiz $8(1): 42-51$

Guo X, Zhang W, Zhong W (2014) Doing topology optimization explicitly and geometrically-a new moving morphable components based framework. J Appl Mech 81(8):081,009

Hecht F (2012) New development in freefem++. J Numer Math 20(34):251-266

Khalil H, Bila S, Aubourg M, Baillargeat D, Verdeyme S, Jouve F, Delage C, Chartier T (2010) Shape optimized design of microwave dielectric resonators by level-set and topology gradient methods. Int J RF Microw Comput-Aided Eng 20(1):33-41

Luo Z, Tong L (2008) A level set method for shape and topology optimization of large-displacement compliant mechanisms. Int $\mathbf{J}$ Numer Methods Engng 76(6):862-892

Luo Z, Tong L, Luo J, Wei P, Wang MY (2009) Design of piezoelectric actuators using a multiphase level set method of piecewise constants. J Comput Phys 228(7):2643-2659

Nishiwaki S, Frecker MI, Min S, Kikuchi N (1998) Topology optimization of compliant mechanisms using the homogenization method. Int J Numer Methods Engng 42:535-559

Nishiwaki S, Min S, Yoo J, Kikuchi N (2001) Optimal structural design considering flexibility. Comput Methods Appl Mech Eng 190(34):4457-4504

Olsson E, Kreiss G (2005) A conservative level set method for two phase flow. J Comput Phys 210(1):225-246

Olsson E, Kreiss G, Zahedi S (2007) A conservative level set method for two phase flow II. J Comput Phys 225(1):785807

Osher S, Sethian JA (1988) Fronts propagating with curvaturedependent speed: Algorithms based on Hamilton-Jacobi formulations. J Comput Phys 79(1):12-49
Park KS, Youn SK (2008) Topology optimization of shell structures using adaptive inner-front (AIF) level set method. Struct Multidiscip Optim 36(1):43-58

Pironneau O (1982) On the transport-diffusion algorithm and its applications to the Navier-Stokes equations. Numer Math 38(3):309332

Pironneau O (1984) Optimal shape design for elliptic systems. Springer, Berlin

Sethian JA, Wiegmann A (2000) Structural boundary design via level set and immersed interface methods. J Comput Phys 163(2):489528

Sigmund O (1997) On the design of compliant mechanisms using topology optimization. Mech Based Des Struct Mechines 25(4):493-524

Sigmund O, Maute K (2013) Topology optimization approaches. Struct Multidiscip Optim 48(6):1031-1055

Sokolowski J, Zochowski A (1999) On the topological derivative in shape optimization. SIAM J Control Optim 37(4):1251-1272

Sokolowski J, Zolesio JP (1992) Introduction to shape optimization. Springer, Berlin

Strain J (1999) Semi-lagrangian methods for level set equations. J Comput Phys 151(2):498-533

Van Dijk NP, Maute K, Langelaar M, Van Keulen F (2013) Levelset methods for structural topology optimization: a review. Struct Multidisc Optim 48(3):437-472

Ville L, Silva L, Coupez T (2011) Convected level set method for the numerical simulation of fluid buckling. Int J Numer Methods Fluids 66(3):324-344

Wang MY, Wang X (2004) Color level sets: a multi-phase method for structural topology optimization with multiple materials. Comput Methods Appl Mech Eng 193(6):469-496

Wang MY, Wang X, Guo D (2003) A level set method for structural topology optimization. Comput Methods Appl Mech Eng 192(1):227-246

Wang S, Wang MY (2006) Radial basis functions and level set method for structural topology optimization. Int J Numer Methods Engng 65(12):2060-2090

Wei P, Wang MY (2009) Piecewise constant level set method for structural topology optimization. Int $\mathbf{J}$ Numer Methods Engng 78(4):379-402

Yamada T, Izui K, Nishiwaki S, Takezawa A (2010) A topology optimization method based on the level set method incorporating a fictitious interface energy. Comput Methods Appl Mech Eng 199(45-48):2876-2891

Zhou S, Li W, Sun G, Li Q (2010) A level-set procedure for the design of electromagnetic metamaterials. Opt Express 18(7):6693-6702

Zhu B, Zhang X, Fatikow S (2015) Structural topology and shape optimization using a level set method with distance-suppression scheme. Comput Methods Appl Mech Eng 283:1214-1239 